Cahiers de géographie du Québec

\title{
Se déplacer pour se soigner. Pratiques et obstacles à Conakry et Douala
}

\author{
Travel for access to health care services. User habits and \\ barriers in Conakry and Douala \\ Desplazarse para curarse. Prácticas y obstáculos en Conakry y \\ Douala
}

\section{Lourdes Diaz Olvera, Didier Plat et Pascal Pochet}

Volume 55, numéro 156, décembre 2011

URI : https://id.erudit.org/iderudit/1008894ar

DOI : https://doi.org/10.7202/1008894ar

Aller au sommaire du numéro

Éditeur(s)

Département de géographie de l’Université Laval

ISSN

0007-9766 (imprimé)

1708-8968 (numérique)

Découvrir la revue

Citer cet article

Diaz Olvera, L., Plat, D. \& Pochet, P. (2011). Se déplacer pour se soigner. Pratiques et obstacles à Conakry et Douala. Cahiers de géographie du Québec, 55(156), 555-573. https://doi.org/10.7202/1008894ar
Résumé de l'article

En matière d'offre de santé en Afrique subsaharienne, les villes apparaissent mieux loties que les villages; aussi, les disparités d'accessibilité aux lieux de soins ont-elles été peu étudiées à l'échelle intra-urbaine. Dans quelle mesure, en contexte de pauvreté, l'accès des citadins africains aux soins se trouve-t-il entravé par les caractéristiques intrinsèques des équipements de santé, mais aussi par leur répartition spatiale et les conditions d'accessibilité ? Des analyses secondaires de deux enquêtes ménages mobilité et d'entretiens réalisés en 2003 à Conakry et à Douala apportent des éléments de réponse. Les problèmes d'accessibilité renforcent l'effet des déficiences propres au secteur sur les difficultés rencontrées pour se soigner et sont plus particulièrement ressentis par les résidants des zones périphériques enclavées, à la fois peu accessibles en transport en commun et sous-équipées. 


\title{
Se déplacer pour se soigner. Pratiques et obstacles à Conakry et Douala
}

\author{
Travel for access to health care services. \\ User habits and barriers in Conakry and \\ Douala
}

\author{
Desplazarse para curarse. Prácticas y \\ obstáculos en Conakry y Douala
}

\author{
Lourdes DIAZ OLVERA, Didier PLAT et \\ Pascal POCHET \\ Laboratoire d'Économie des Transports, \\ Université de Lyon \\ Lourdes.Diaz-Olvera@entpe.fr \\ Didier.Plat@entpe.fr \\ Pascal.Pochet@entpe.fr
}

\section{Résumé}

En matière d'offre de santé en Afrique subsaharienne, les villes apparaissent mieux loties que les villages; aussi, les disparités d'accessibilité aux lieux de soins ont-elles été peu étudiées à l'échelle intra-urbaine. Dans quelle mesure, en contexte de pauvreté, l'accès des citadins africains aux soins se trouve-t-il entravé par les caractéristiques intrinsèques des équipements de santé, mais aussi par leur répartition spatiale et les conditions d'accessibilité? Des analyses secondaires de deux enquêtes ménages mobilité et d'entretiens réalisés en 2003 à Conakry et à Douala apportent des éléments de réponse. Les problèmes d'accessibilité renforcent l'effet des déficiences propres au secteur sur les difficultés rencontrées pour se soigner et sont plus particulièrement ressentis par les résidants des zones périphériques enclavées, à la fois peu accessibles en transport en commun et sous-équipées.

\section{Mots-clés}

Santé, accessibilité, mobilité quotidienne, pauvreté, Conakry, Douala.

\begin{abstract}
Sub-Saharan African cities are believed to be better served than villages with regard to access to health care. Yet differences at the intra-urban level have seldom been analyzed. Fundamental questions remain as to the extent to which, in a context of urban poverty, access by Africans to health care is affected both by factors intrinsic to the health sector itself as well as by the spatial distribution of health care providers and accessibility conditions. The aim of this paper is to analyze accessibility to health care services. Empirical evidence from two household travel surveys and interviews carried out in 2003 in Conakry and Douala suggests that accessibility barriers reinforce the negative effects of deficiencies specific to the health care sector. This situation is even more problematic in the case of peripheral areas, with their limited access to public transport and to the services and infrastructures characteristic of larger urban communities.
\end{abstract}

\section{Keywords}

Health care, accessibility, daily travel, poverty, Conakry, Douala. 


\section{Resumen}

La oferta de servicios de salud en Africa subsahariana es mayor en las ciudades que en los pueblos y por lo tanto las desigualdades de acceso a los lugares de asistencia médica son muy poco estudiadas a escala intra-urbana. ¿Cómo el acceso de los ciudadanos africanos a tales servicios, en un contexto de pobreza, se encuentra obstaculizado por las características intrínsecas de los establecimientos de salud, de su distribución espacial y de sus condiciones de accesibilidad? Los análisis secundarios de dos encuestas en los hogares sobre la movilidad cotidiana y de entrevistas realizadas en 2003 en Conakry y Douala proporcionan elementos de respuesta. Los problemas de accesibilidad acentúan las deficiencias del sector, complicando el recurso a los servicios de salud. Esta situación es aún más problemática para los residentes de las zonas periféricas enclavadas, poco accesibles por transporte público y con pocas infraestructuras.

\section{Palabras claves}

Salud, accesibilidad, movilidad cuotidiana, pobreza, Conakry, Douala.

\section{Introduction}

Les politiques publiques de réduction de la pauvreté des populations des pays du Sud mettent en avant le renforcement de leurs «capacités» pour améliorer leurs capitaux économique, social, humain. Un tel renforcement passe notamment par un accès facilité à la santé. Mais le contexte politique et économique des dernières décennies a rendu difficile la mise en œuvre des politiques publiques de santé, ce qui laisse craindre que, comme dans d'autres secteurs, les objectifs de 2015 du Millénaire pour le développement ne puissent être atteints (Sahn et Stifel, 2003).

Depuis les années 1990, la carte sanitaire de nombre de pays subsahariens s'est déployée de manière hiérarchisée sur le territoire national, avec comme objectifs affichés le développement des premiers soins et de la prévention. Parallèlement, le secteur privé a sensiblement accru sa présence, souvent d'ailleurs à travers des processus de «siphonnage» du secteur public (Olivier de Sardan et al., 2007). Néanmoins, si des efforts considérables ont été entrepris en faveur de la desserte en milieu rural, les faibles densités rendent difficile la localisation de centres de santé à proximité des villages et communautés, au-delà des soins primaires. L'offre demeure concentrée dans les ensembles urbains, notamment dans les grandes villes au sein desquelles les possibilités d'accès à la santé sont théoriquement démultipliées par la diversité de l'offre de services de santé. Pourtant, avec la poursuite de l'urbanisation à un rythme soutenu dans les pays du Sud, et particulièrement en Afrique subsaharienne, la santé des populations urbaines devient un enjeu central, non seulement de politique sectorielle, mais aussi plus largement dans les problématiques de développement (Harpham, 2009).

L'existence d'une offre sanitaire ne garantit pas qu'elle soit utilisée. Encore faut-il qu'elle soit financièrement abordable, physiquement accessible et de qualité suffisante pour répondre aux besoins et aux attentes de ses usagers potentiels. Au Sud, mais 
également dans les villes du Nord, les obstacles à la fréquentation des services de santé affectent différemment les pratiques sanitaires des populations urbaines selon leur localisation résidentielle et leur situation socioéconomique et entraînent de fortes inégalités d'accès aux soins, au détriment des plus défavorisés. En particulier, les disparités spatiales dans la répartition des services de santé et les capacités diverses qu'ont les populations de se rendre aux lieux de soins font de l'accessibilité un élément clé d'analyse des conditions d'équité dans l'accès aux soins (Guagliardo, 2004).

L'accessibilité peut être définie, de façon générale, comme «offre de mobilité, ensemble des possibilités effectives de relier deux lieux par un déplacement» (Lévy et Lussault, 2003 : 35). Elle permet d'évaluer le potentiel de services utilisables sur un territoire donné et ne renseigne donc pas sur l'accès effectif à ces services, dont elle est l'une des conditions (Penchansky et Thomas, 1981). L'accessibilité est appréhendée en prenant en compte la localisation de l'offre de services de santé disponibles sur un territoire ou une aire donnés et la distance (ou le temps de transport) nécessaire pour s'y rendre. Selon les choix de mesures faits et les données à disposition, l'accessibilité spatiale se traduit par une grande diversité d'indicateurs (Raux et al., 2008) : indicateurs d'offre et de la répartition de l'offre (nombre de services au sein d'une zone ou d'un rayon donné autour d'un point), de distance des lieux de résidence à un ou à plusieurs services de santé (à vol d'oiseau, rectilinéaire, par affectation sur un réseau), de temps de trajet (global ou en intégrant la disponibilité de transport public ou individuel), de coûts (sommes à payer pour se rendre au lieu de soins), voire de coûts ou de temps généralisés. La prise en compte des espaces d'activités, à partir des pratiques de déplacement dans la ville, permet d'appréhender plus finement les possibilités d'accès aux soins individuels (Sherman et al., 2005) ; de même, la prise en compte des migrations alternantes modifie l'accessibilité spatiale des actifs aux magasins alimentaires et aux équipements sportifs, et ce, de façon différenciée selon le lieu de résidence (Salze et al., 2011). Les options méthodologiques ne sont pas neutres et peuvent contribuer à influencer la nature des conclusions tirées quant à l'impact de l'accessibilité sur l'accès aux soins (Talen et Anselin, 1998; Apparicio et al., 2008). Toutefois, un certain constat paraît se dégager quant au déficit d'accessibilité dans les périphéries des villes. Ainsi, à partir de mesures intégrant les caractéristiques de mobilité et les difficultés de transport, Paez et al. (2010) montrent que les résidants âgés des espaces périurbains de Montréal connaissent une accessibilité relativement faible aux équipements de santé, et ce, même lorsqu'ils sont motorisés. Des études menées sur les pays du Sud mettent également en lumière le déficit d'accessibilité aux services de santé dans les zones urbaines périphériques et à habitat spontané, ainsi que l'impact de la distance et des difficultés de transport sur la fréquentation (Eser et al., 2005 dans le cas d'une ville turque moyenne, Bhanderi et Kannan, 2010 à Rajkot, ville indienne; More et al., 2011 à Mumbai).

Dans les villes africaines, le sous-équipement sanitaire, l'allongement continu des distances lié à l'urbanisation galopante, les carences des infrastructures routières ainsi que les déficiences et le coût du transport urbain pour les ménages (Diaz Olvera et al., 2008) posent la question des contraintes d'accessibilité aux équipements. Différentes études pointent le sous-équipement sanitaire des quartiers périphériques, généralement pauvres et d'habitat non loti, et identifient l'importance de l'accessibilité parmi les difficultés d'accès aux soins dans les périphéries des villes (Salem, 1998; Mbara, 2002 à Harare; Dramé, 2006 dans le cas de Dakar; Cadot et Harang, 2006 à 
Ouagadougou; Fotso et al., 2008 concernant l'usage des soins prénataux dans les bidonvilles de Nairobi ; Essendi et al., 2010 sur les urgences obstétriques à Nairobi ; Sasaki et al., 2010 à Lusaka). Toutefois, une étude plus ancienne sur Ouagadougou (Develay et al., 1996) concluait à une absence d'effet de la distance aux services de soins sur leur utilisation. Ce résultat dissonant laisse ouverte l'hypothèse d'une possible dégradation de la situation dans cette ville depuis la fin des années 1980 du fait de la poursuite d'une forte croissance urbaine.

Les différentes études sur l'accessibilité des lieux de soins sont pour la plupart menées dans le champ des études sur la santé, et si la mobilité et les pratiques de déplacement font l'objet d'un intérêt croissant, les usages des transports urbains et, plus largement, les pratiques de mobilité quotidienne restent insuffisamment analysés à l'aune de l'accessibilité aux soins. Pourtant, de précédents travaux issus d'enquêtes sur la mobilité ont mis en évidence que les difficultés de déplacement au quotidien pèsent fortement sur les possibilités d'accès aux aménités urbaines en Afrique subsaharienne, par exemple en ce qui concerne l'emploi ou l'éducation (Diaz Olvera et al., 2007 et 2010). Dans cette perspective, l'objectif de cet article est d'analyser dans quelle mesure et selon quelles modalités l'accessibilité spatiale aux services de santé constitue un obstacle à l'accès aux soins, entendu ici au sens d'utilisation effective des services de santé. Pour ce faire, des analyses comparées de données individuelles quant à la mobilité des citadins, au type de services de santé fréquenté et aux obstacles rencontrés, sont menées sur Conakry (Guinée) et Douala (Cameroun). Ces deux villes de taille assez proche (de l'ordre respectivement de 1,7 et 2 millions d'habitants à la date des enquêtes) ont l'intérêt d'offrir des situations contrastées quant au système de transport, moins développé à Conakry tant en mode individuel que collectif, et quant au système de soins, le secteur privé occupant une place plus réduite à Conakry (Gobbers, 2004).

L’article est structuré en quatre parties. Nous présentons d'abord le contexte dans lequel l'offre sanitaire est assurée en Afrique et les données utilisées. Nous resituons ensuite les spécificités des déplacements urbains pour la santé dans les pratiques et les conditions de réalisation des déplacements à Conakry et à Douala. Puis, après avoir présenté les pratiques déclarées des différents types de services de santé, nous abordons la question de l'accessibilité, par la voie de la distance estimée du domicile au service et des difficultés rencontrées pour s'y rendre, en la mettant en perspective avec d'autres obstacles ressentis dans l'accès aux soins dans les deux villes. Enfin, en conclusion, nous présentons quelques implications de ces résultats quant au rôle de l'accessibilité spatiale dans les difficultés d'accès aux soins rencontrées en milieu urbain africain.

\section{Une offre de santé en profonde évolution}

En matière de politique de santé, les deux dernières décennies ont été marquées par les réformes issues de l'Initiative de Bamako, lancée en 1987. Cette politique visait à redynamiser l'offre à travers l'extension du réseau des soins de santé primaires, la participation communautaire dans la gestion des centres, le recouvrement partiel des coûts et la disponibilité de médicaments génériques (Ridde, 2005). Le financement partiel des services de santé par les usagers au moyen du «recouvrement des coûts» a ouvert la voie à une privatisation partielle du service et s'est traduit par des coûts plus élevés pour la plupart des ménages. C'est dans ce cadre que les systèmes de santé 
se sont restructurés, dès 1988 en Guinée, à partir des années 1990 au Cameroun, en prenant appui sur les districts de santé comme unité opérationnelle, bases d'une hiérarchisation des structures sanitaires. Ces centres et postes de santé ont pour objectif premier d'offrir un ensemble minimum d'activités (soins ambulatoires, prévention, accouchements). Ils sont définis généralement selon un seuil démographique, auquel peuvent s'ajouter d'autres critères, socioculturels ou géographiques, voire politiques, notamment pour le Cameroun (Okalla et Le Vigouroux, 2001).

À Douala et à Conakry, comme dans d'autres contextes (voir par exemple, Mamadani et Bangser, 2004 pour la Tanzanie), ces réformes se sont heurtées à de multiples problèmes : décentralisation désordonnée (Médard, 2001), renforcement des pratiques de corruption au sein du personnel de santé (Jaffré, 2004), insuffisante prise en compte de l'offre privée et confessionnelle dans l'élaboration de la carte sanitaire alors que l'offre confessionnelle assure $40 \%$ des soins au Cameroun (Gauvrit et Okalla, 2001), «balkanisation des aides internationales qui se partagent le territoire» (Médard, 2001 : 3 ) en tenant peu compte des offres existantes et des besoins réels. Ce relatif échec des réformes sectorielles peut également s'expliquer par un engagement budgétaire limité de la part des États. Ainsi, en 2008, la santé a représenté 7,9\% de la dépense publique totale au Cameroun et $4,7 \%$ en Guinée (OMS, 2010) et les dépenses de santé sont couvertes principalement par la dépense privée: $72 \%$ au Cameroun et $89 \%$ en Guinée, assurée en quasi-totalité par les ménages (respectivement à 95\% et 99\%).

Alors qu'une toute petite minorité de citadins bénéficie de l'assurance maladie - de l'ordre de 1\% en Guinée (Doumbouya, 2008) et de $3 \%$ au Sénégal, (Fall, 2007) -, le recouvrement des coûts ainsi que les pratiques de corruption d'une partie du personnel de santé pénalisent particulièrement les populations les plus vulnérables: il faut disposer d'argent pour obtenir une consultation et plus encore pour l'achat des médicaments, voire de certains accessoires médicaux. L'automédication et diverses formes de médecine traditionnelle apparaissent alors comme des recours possibles en cas de manque de moyens, mais elles renvoient aussi à d'autres déterminants tels que le poids de l'environnement familial et de l'habitude ou la minimisation des symptômes ressentis, qui expliquent leur diffusion bien au delà des seules catégories les plus pauvres (Brunet-Jailly, 2001 ; Commeyras et al., 2006). Elles présentent également l'avantage, tout particulièrement l'automédication, de pouvoir se pratiquer dans le quartier, comme le déclare ce jeune Conakryka inactif: «Parfois, j’ai des maux de tête, mais dans ce cas j'achète des comprimés chez le tablier du coin.»

En effet, à la contrainte monétaire, s'ajoutent les effets de l'inégale répartition des équipements de santé au sein des villes. Ainsi, les deux hôpitaux nationaux et plus de la moitié de leur personnel de santé sont situés à Conakry (Comolet, 2000) qui représente un cinquième de la population guinéenne, mais les périphéries de la capitale de la Guinée demeurent sous-équipées. À la différence des communes centrales de la ville, les communes périphériques, très vastes et très peuplées, ne disposent pas d'hôpital; elles sont également sous-équipées en centres de santé (un pour 60000 habitants environ contre un pour 10000 à un pour 25000 habitants dans les communes centrales) (Groupe Huit-Bceom, 2003).

À Douala, la répartition spatiale des établissements n’est guère moins déséquilibrée, les seules différences notables étant l'existence d'hôpitaux de districts, mieux dotés en personnel et mieux équipés que les centres locaux, et la forte présence d'établis- 
sements privés, notamment confessionnels. Mais les soins importants nécessitent de se rendre dans l'un des deux hôpitaux publics, tous deux proches du centre. Dans les deux villes, les résidants des zones périphériques apparaissent ainsi relativement à l'écart de l'offre sanitaire, ce qui peut entraver leur recours aux services de santé.

Si elle n'est pas seule en jeu dans l'accès aux soins, la question de l'accessibilité aux structures sanitaires se pose avec acuité dans les villes africaines. Comment les citadins parviennent-ils, ou non, à assurer leurs besoins de santé, compte tenu d'une offre inégalement répartie dans l'aire urbaine et présentant d'importantes différences de qualité de service et de coût d'usage?

\section{Données et indicateurs analysés}

Pour tenter de répondre à cette question, nous nous appuyons principalement sur des analyses secondaires de deux enquêtes quantitatives et d'entretiens réalisés en 2003 dans le cadre d'une étude sur la mobilité quotidienne et les conditions d'accès des populations pauvres aux équipements urbains à Douala et à Conakry (Sitrass, 2004a et b).

Dans chaque ville, l'enquête par questionnaire a été réalisée auprès d'environ 600 ménages, et les entretiens, auprès d'une trentaine de citadins pauvres.

Dans chaque ville, les enquêtes ont été menées dans 30 quartiers caractérisés par une surreprésentation des populations pauvres. Chaque échantillon a été redressé vis-à-vis de grandes enquêtes représentatives menées dans la ville (respectivement les enquêtes UNICEF 1999 et QUIBB 2002, et les enquêtes sur les dépenses des ménages de Yaoundé et Douala 2000, ECAM II 2001 et CAVIE 2002). Le redressement de chaque échantillon a intégré la localisation et le niveau de revenus, de façon à assurer la comparabilité et la représentativité des résultats qui en sont issus (Sitrass, 2004a et b).

Le questionnaire de l'enquête ménages comprend trois niveaux d'information: le ménage, l'individu de plus de 10 ans et la mobilité de la veille du jour d'enquête pour chacun de ces individus (2700 à Conakry, près de 1900 à Douala). En vue de préserver la comparabilité des résultats avec des enquêtes antérieures de méthodologie proche, les résultats sur la mobilité présentés dans cet article portent sur les 15 ans et plus, soit 2345 individus à Conakry et 1701 à Douala. Les informations classiques de positionnement démographique et socioéconomique des ménages et des individus ont été recueillies.

Le questionnaire renseigne notamment sur l'accès des ménages aux équipements de base dont la santé, en distinguant les deux niveaux principaux du système de soins (dispensaire, hôpital) et le caractère public ou privé du service à partir de la fréquence d'usage déclarée (oui souvent/oui parfois/non jamais). Une seule personne (la personne de référence ou son conjoint) répondait pour l'ensemble du ménage. Le croisement des deux niveaux de service et de leur nature publique ou privée met en évidence que trois ménages sur dix à Conakry et plus d'un sur deux à Douala n'utilisent qu'un seul type, et ce, en dépit de tailles de ménages généralement importantes. Ces taux incitent à distinguer, pour mettre en évidence des pratiques d'accès aux soins différenciées socialement et spatialement, différents types d'usage des services de 
santé: usage du dispensaire public seulement, usage de l'hôpital public seulement, usage des deux niveaux publics, usage du secteur privé, usage mixte (au moins un établissement public et un établissement privé) et, enfin, absence d'usage.

Les autres informations recueillies quant à l'accès aux équipements de santé concernent la localisation de l'établissement, le mode de transport utilisé, le temps d'accès, ainsi que la nature des obstacles éventuels rencontrés dans sa fréquentation. Pour chacun des quatre types d'établissement, centres de santé et hôpitaux, publics et privés, huit modalités étaient proposées aux ménages $(1:$ trop éloigné, 2 : problème de transport, 3 : trop cher, $4:$ attente trop longue, $5:$ mauvaise qualité du service, 6 : insuffisance spécialité, $7:$ salles surchargées, $8:$ autre problème, préciser, 9: pas de problème rencontré), parmi lesquelles trois au plus pouvaient être choisies. L'accessibilité est considérée comme un obstacle si l'individu a cité au moins l'une des deux premières modalités, la qualité de service s'il a cité au moins l'une des modalités 4 à 7 , et le coût s'il a cité la modalité 3 . Enfin les réponses mentionnant un «autre problème» ont été affectées à l'une des trois catégories précédentes en fonction de la précision apportée.

Ces enquêtes appréhendent la mobilité de chaque membre du ménage par l'ensemble des déplacements qu'il a réalisés la veille du jour de l'enquête, du lundi au samedi. Chaque déplacement est repéré par une origine et une destination, une durée, un ou plusieurs modes de transport, l'activité finale. Cette dernière s'appuie sur une nomenclature de motifs, dans laquelle apparaît explicitement la santé. La mise en commun des déplacements réalisés permet d'offrir une photographie de la mobilité, un jour moyen de semaine.

Les entretiens étaient centrés sur les pratiques et conditions de mobilité de la personne et d'accès aux aménités de base dont les services de santé, ainsi que sur ses perceptions (Sitrass, 2004a et b). Les personnes sondées résidaient dans des quartiers divers des deux villes et avaient en commun de disposer de bas ou de très bas revenus.

\section{Accès aux soins et mobilité quotidienne}

L'analyse des déplacements d'un jour moyen apporte un premier éclairage des pratiques de déplacement liées à la santé. Comparée aux motifs professionnels, aux études ou encore à la sociabilité, la santé fait logiquement figure d'activité exceptionnelle, générant environ $1 \%$ du total des déplacements dans la ville. Toutefois, une part des déplacements qui lui sont liés échappe à ce décompte: l'achat de médicaments (classé au motif Achat), l'accompagnement d'un malade (motif Accompagnement), ainsi probablement que certains déplacements liés à la médecine traditionnelle (déclarés comme Visites).

Les spécificités des déplacements liés à la santé apparaissent clairement sur le premier plan d'une analyse factorielle des correspondances multiples (AFCM) croisant le motif de déplacement, le mode, la destination et la durée (figure 1). Nous nous focalisons ici sur la seule ville de Conakry, car le même lien étroit entre les variables, révélé par la forme parabolique du nuage, apparaît pratiquement à l'identique à Douala. 


\section{Figure 1 Les spécificités des déplacements pour le motif santé dans la mobilité des Conakrykas}

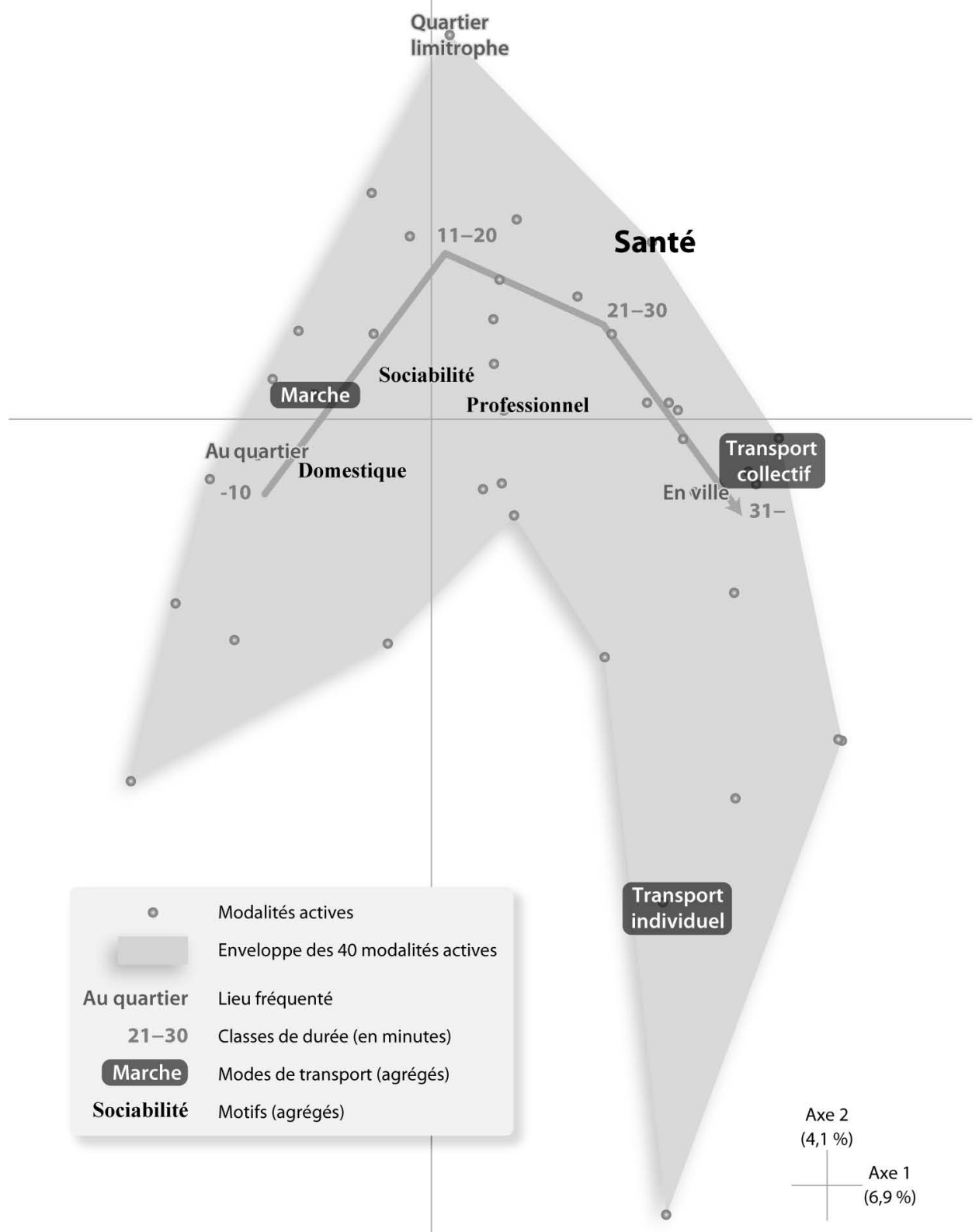

Premier plan de l'AFCM croisant le lieu, le motif, le mode de transport et la durée de déplacement. Sauf pour le lieu et le motif Santé, ne sont précisées sur la figure que les agrégations des modalités des variables actives, pour des raisons de lisibilité:

- Classes de durée (mn). -10: 1-5, 6-10; 11-20: 11-15, 16-20; 21-30:21-25, 26-30; 31-: 31-45, 46-60, 60-.

- Modes de transport. Marche: marche; Transport collectif: minibus, taxi; Transport individuel: voiture, deux-roues.

- Motif (21 modalités initiales). Domestique: approvisionnement en eau, achats alimentaires, autres achats, accompagnements,

démarches, santé...; Professionnel: travail, école, recherche de travail...; Sociabilité: visite à des parents, à des voisins, à des amis,

loisirs..

Source: Enquête ménages mobilité Conakry, 2003. 
À l'exception du motif santé, n'apparaissent explicitement sur la figure que des regroupements des modalités initiales. La santé s'apparente aux activités domestiques permettant le fonctionnement et la reproduction du ménage, comme l'approvisionnement en eau, les achats alimentaires ou autres, généralement effectuées à proximité du domicile et à pied. Pour autant, elle s'en distingue très sensiblement puisqu'elle apparaît sur la figure dans une position excentrique, à l'exact opposé, ce qui traduit la nécessité d'un éloignement du domicile et, corrélativement, des temps de déplacement assez importants et un relatif délaissement de la marche.

La répartition modale des déplacements pour la santé est similaire dans les deux villes (figure 2). Compte tenu des faibles niveaux d'équipement en véhicules particuliers, les transports collectifs se taillent la part du lion. Utiliser les transports en commun signifie généralement un déplacement long (30 min à Douala, 37 min à Conakry). Ces déplacements ne sont pas des plus aisés à entreprendre lorsqu'on est malade, compte tenu des taux de remplissage généralement élevés des véhicules, des longs temps d'attente, des trajets pédestres terminaux ou entre deux correspondances, à l'image d'observations faites à Oran (Rebouha, 2007). Destination inhabituelle, limitations physiques, éloignement du service, nombre d'éléments justifient l'usage des transports en commun, même lorsque les moyens financiers sont limités. En conséquence, la marche est notablement moins fréquente que pour d'autres activités, dans des villes où nombre de citadins «ne comptent que sur leurs pieds» (Kinda, 1987: 491). Dans l'ensemble des déplacements réalisés, la marche est utilisée par les 15 ans et plus à hauteur de $71 \%$ des déplacements à Conakry et de $60 \%$ à Douala. Signe que l'usage des transports motorisés ne va pas de soi, un jour donné, une part importante des citadins n'utilise aucun mode mécanisé. C'est le cas de près de 4 Doualais sur 10, soit parce qu'ils se sont déplacés exclusivement à pied (29\%), soit parce qu'ils ne se sont pas déplacés du tout ( $8 \%$ ). À Conakry, plus de la moitié des citadins de 15 ans et plus n’utilisent aucun mode de transport mécanisé un jour donné (dont $44 \%$ de «marcheurs exclusifs» et $11 \%$ de non-mobiles). Les espaces fréquentés au quotidien portent la marque de cette faible mobilité mécanisée, avec une prédominance de déplacements dans le quartier de résidence ou dans les quartiers limitrophes, et une fréquentation limitée du centre administratif et commercial de la ville (effective pour seulement $22 \%$ des plus de 15 ans un jour donné à Douala, et même $5 \%$ à Conakry). Rares sont les citadins des deux villes qui peuvent profiter d'une sortie vers le centre-ville pour fréquenter un service de santé.

Plus précisément, les ménages résidant dans un quartier enclavé, mal desservi par les transports collectifs (à plus de 15 min à pied de l'arrêt le plus proche), sont plus nombreux à se rendre à pied au lieu de soins que les autres ( $40 \%$ contre $22 \%$ à Douala; $68 \%$ contre $22 \%$ à Conakry). La marche est notamment utilisée par une partie des citadins pour atteindre des équipements situés au delà du voisinage. La durée moyenne des déplacements pédestres est de 13 minutes à Douala, mais dépasse 20 minutes à Conakry, soit, à une vitesse minimale de $3 \mathrm{~km} / \mathrm{h}$, une distance moyenne entre domicile et lieu de soins de l'ordre du kilomètre. Mais la proximité ou l'éloignement du lieu de soins dépend également du type d'équipement. 
Figure 2 Répartition des modes de transport utilisés pour les déplacements pour la santé et pour l'ensemble des déplacements, à Conakry et à Douala (\%)

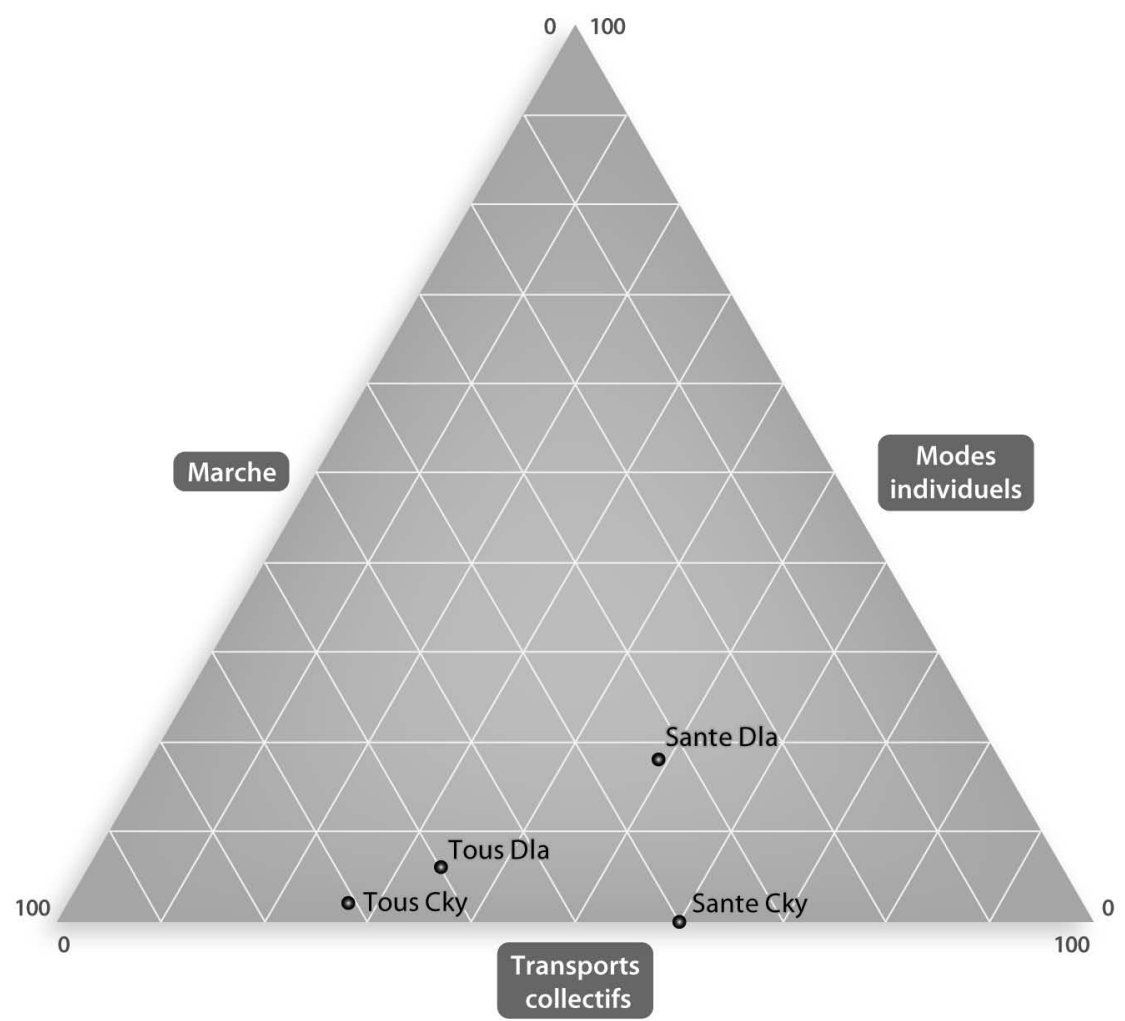

Source: Enquêtes ménages mobilité, individus de 15 ans et plus

\section{Équipements fréquentés, inégalités d'accès aux soins et difficultés d'accessibilité}

Pour appréhender la part prise par les difficultés d'accessibilité dans l'accès aux soins, nous lions d'abord les différentes configurations de fréquentation des équipements sanitaires aux caractéristiques des ménages. Nous mettons ensuite en perspective les difficultés d'accessibilité perçues par rapport aux autres freins à l'accès aux équipements, avant d'examiner les difficultés d'accessibilité selon le quartier de résidence.

\section{Types de fréquentation des établissements de soins et caractéristiques du ménage}

La prise en compte de la nature publique ou privée des services fréquentés par les ménages et de leur place dans la hiérarchie du système de soins fait émerger des usages assez différents d'une ville à l'autre (figure 3). Sous ses différentes formes, le recours exclusif au public se révèle nettement plus répandu à Conakry (70\% des ménages) qu'à Douala (à peine 40\%). Corrélativement, à Douala, l'usage exclusif du secteur privé 
et la forme mixte (usage du privé et du public) sont, à l'inverse, bien plus présents, respectivement 24 et $36 \%$, contre à peine 5 et $25 \%$ dans la capitale guinéenne. Ces différences reflètent des effets d'offre, à savoir la moindre pénétration du secteur privé à Conakry. Mais elles renvoient également à des structures sociodémographiques des ménages distinctes. Dans les deux villes, les ménages de plus grande taille tendent à fréquenter plusieurs catégories d'équipement, du fait d'une hétérogénéité des besoins sanitaires de leurs membres, mais aussi des stratégies de diversification comme on en observe avec l'école (Diaz Olvera et al., 2010). L'écart notable de taille entre ménages conakrykas et doualais (7,2 personnes contre 4,2) explique le choix sensiblement moins fréquent d'une structure unique dans la capitale guinéenne.

\section{Figure 3 Services de santé fréquentés par les ménages}

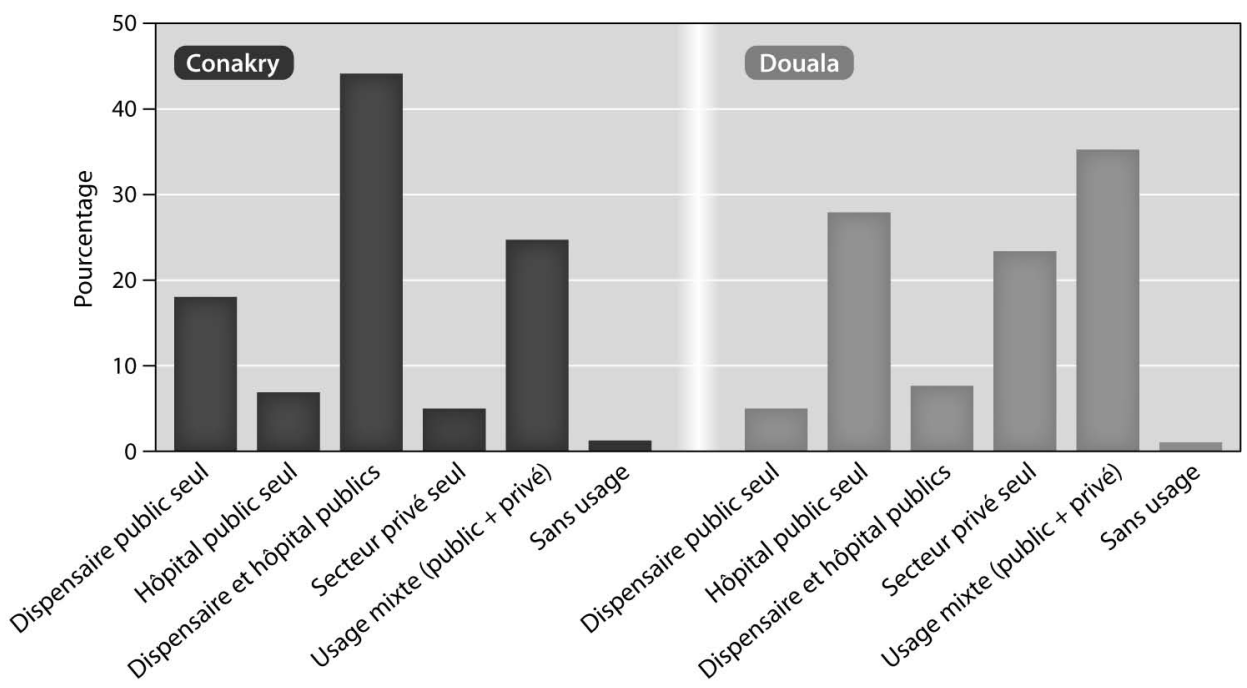

Source: Enquêtes ménages mobilité 2003.

Le type de fréquentation dépend également des ressources du ménage. Un niveau de vie plus élevé se traduit par une moindre fréquentation des structures de base publiques et privées (figure 4). Cette désaffection s'accompagne à Conakry d'un accroissement du recours aux pratiques mixtes, et à Douala d'un usage exclusif de l'hôpital public plus répandu. L'effet de la contrainte économique apparaît également à travers d'autres indicateurs. Les ménages doualais estimant que leur situation économique s'est dégradée au cours de l'année, de même que ceux dont la personne de référence est une femme, privilégient le recours aux établissements publics.

\section{Accessibilité et autres obstacles à la fréquentation des services de santé}

L'analyse des obstacles perçus par les ménages complète l'examen des difficultés d'accès aux services de santé. Elle permet de resituer l'importance de l'accessibilité (éloignement, problèmes de transport) à côté du coût et de la qualité de service (services surchargés, longues attentes, manque de matériel, etc.). 
Figure 4 Services de santé fréquentés selon le niveau de vie du ménage

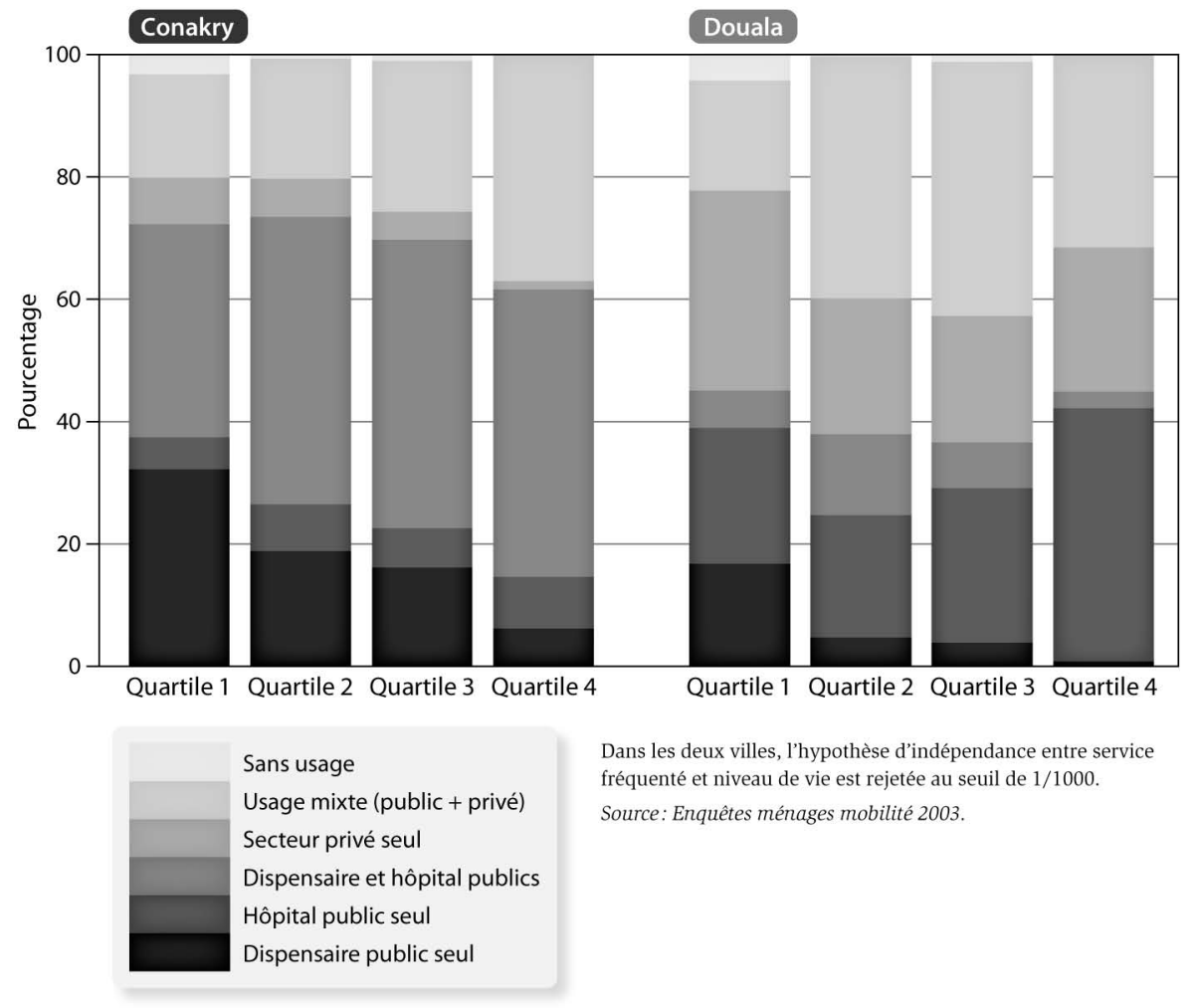

Le poids relatif des obstacles cités par les ménages varie en fonction du type d'établissement (figure 5). La mauvaise qualité des soins est très majoritairement ressentie comme un problème. À Douala comme à Conakry, cette qualité insuffisante concerne l'ensemble des structures publiques, hôpitaux compris. Elle constitue également la critique la plus fréquemment adressée aux dispensaires privés à Douala, contrepartie probable de leur diffusion spatiale accélérée et sans contrôle. À l'inverse, les structures donnant les meilleures garanties de qualité sont considérées comme trop coûteuses par les ménages, cliniques et hôpitaux privés des deux villes et dispensaires privés à Conakry, vis-à-vis desquels plus des trois quarts et jusqu'à $90 \%$ des ménages mentionnent la barrière du coût. De tels chiffres laissent entrevoir que ces services sont de fait inutilisables par la très grande majorité des citadins.

L’arbitrage obligé entre qualité et coût des soins ressort également, dans les entretiens: «Le centre de Bonassama, il faut prendre soit le bendskin [moto-taxi] soit le taxi pour s'y rendre. [...] On va souvent à Bonassama parce que là-bas il y a quand même de bons médecins alors que dans les centres ce sont les infirmiers. Quand on n'a pas les moyens on va dans les centres qu'il y a dans les quartiers» (vendeuse doualaise, 32 ans, habitant à près de $3 \mathrm{~km}$ du centre de soins de Bonassama). Établissement public ou privé, le coût des soins est l'élément clé du choix, dès lors qu’un choix paraît possible: "Quand vous arrivez à l'hôpital, vous n'êtes pas bien reçu. [...] alors que dans les centres de santé on vous demande de l'argent qui entre dans le traitement, là-bas 
Figure 5 Obstacles à la fréquentation des services de santé (\% des ménages les citant)*
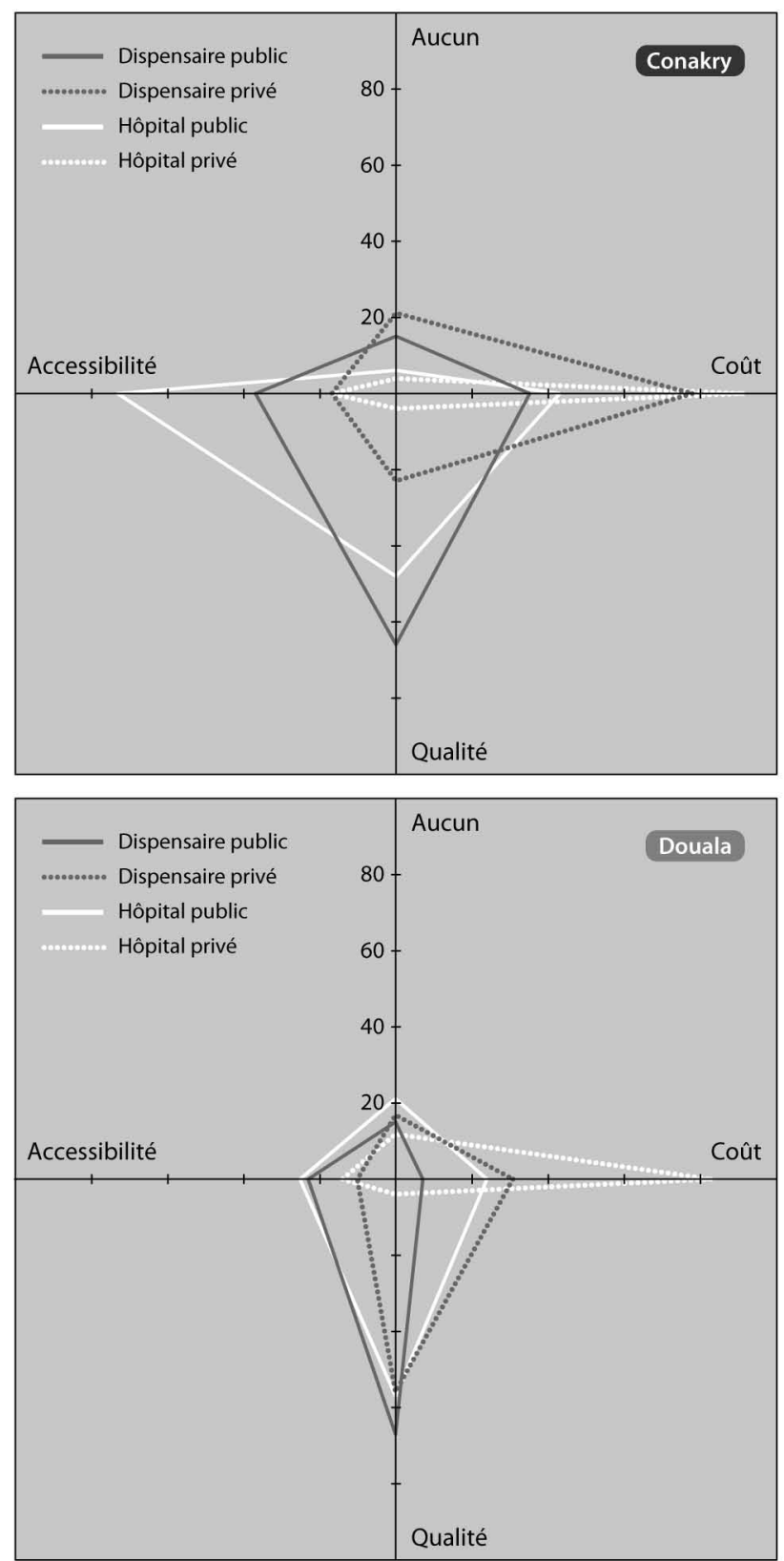

* L'accessibilité est mentionnée comme obstacle si l'individu a cité au moins l'un des deux problèmes suivants: l'éloignement du service/des problèmes de transport pour s'y rendre. L'accessibilité est significativement (au seuil de $5 \%$ ) plus citée à Conakry qu'à Douala pour les établissements publics, mais au même seuil, la différence n'est pas significative pour les établissements privés.

Lecture du graphique: à Douala, $21 \%$ des ménages déclarent n’avoir aucun problème d'accès à l'hôpital public.

Source: Enquêtes ménages mobilité 2003. [à l'hôpital] il faut d'abord donner... mouiller la barbe de ceux qui sont les infirmiers avant de payer l'argent qui entre dans les caisses de l’État» (lycéenne doualaise, 23 ans). Un service comme l'hôpital, souvent éloigné géographiquement, paraît alors également très loin dans le champ des possibles envisagés pour se soigner. La faible qualité de service des institutions publiques, très souvent évoquée pour les structures de proximité, à l'instar des exemples très révélateurs donnés par Fall (2007) dans le cas des ménages pauvres de Dakar, explique très largement leur mauvaise image. En limitant l'univers de choix, la mauvaise qualité de service contribue à dégrader l'accès effectif au système de soins.

\section{Accessibilité aux soins et enclavement du quartier de résidence}

$\mathrm{Au}$ vu de la localisation des structures fréquentées, c'est le dispensaire privé qui apparaît le plus souvent comme la structure de proximité, à Conakry comme à Douala : deux ménages sur trois s'y rendent dans leur quartier d'habitation, alors que ce n'est le cas que de deux sur cinq pour le centre public. Ce constat est confirmé par une estimation des distances parcourues pour se rendre au(x) dispensaire(s) et centre(s) de santé fréquenté(s). Celles-ci sont calculées à 
partir des durées de trajet déclarées, en prenant par convention comme vitesse pédestre, une vitesse relativement basse de $3 \mathrm{~km} / \mathrm{h}$ et comme vitesse en transport collectif, $10 \mathrm{~km} / \mathrm{h}$. Il s'agit donc de distances approximatives et minimales, mais leur hiérarchisation demeurerait identique en considérant des vitesses plus élevées. Le dispensaire public «utile», au sens où il suscite des usages effectifs, n’apparaît pas comme le service le plus proche (tableau 1). À Douala, il est situé en moyenne 600 $\mathrm{m}$ plus loin du domicile que le dispensaire privé, l'écart étant plus réduit (et non significatif) à Conakry (de l'ordre de $200 \mathrm{~m}$ ).

À Conakry plus encore qu'à Douala, la proximité des équipements de proximité est toute relative, d'autant que certains d'entre eux sont évités par des usagers contraints d'arbitrer entre distance et coût ou qualité du service, à l'image de cette vendeuse résidant dans un quartier de la périphérie de Conakry, qui dit: «Je vais au centre de santé de Yimbaya [à 2,5 km] car le coût est trop élevé dans notre quartier.» Cela confirme des pratiques observées au Tchad (Gauthier et Wane, 2008).

\section{Tableau 1 Distances estimées du domicile au centre de proximité fréquenté, selon la desserte du quartier par les transports en commun $(\mathbf{k m})^{\mathrm{a}}$}

\begin{tabular}{|c|c|c|}
\hline & \multicolumn{2}{|c|}{ Tous usages } \\
\hline & Public & Privé \\
\hline Conakry & 2,3 & 2,1 \\
\hline Sans desserte TC & 2,5 & 2,6 \\
\hline Avec desserte TC & $\begin{array}{l}2,2 \\
\text { ns }\end{array}$ & $\begin{array}{c}1,5 \\
*\end{array}$ \\
\hline Douala & 1,9 & 1,3 \\
\hline Sans desserte TC & 2,1 & 1,8 \\
\hline Avec desserte TC & $\begin{array}{l}1,8 \\
\text { ns }\end{array}$ & $\begin{array}{c}1,1 \\
*\end{array}$ \\
\hline \multicolumn{3}{|c|}{$\begin{array}{l}\text { a: Sans desserte TC: À } 15 \text { min à pied ou plus de l'arrêt de transport collec } \\
\text { le plus proche. }\end{array}$} \\
\hline \multicolumn{3}{|c|}{$\begin{array}{l}\text { Avec desserte TC: À moins de } 15 \text { min à pied d'un arrêt de transport } \\
\text { collectif le plus proche. }\end{array}$} \\
\hline \multicolumn{3}{|c|}{$\begin{array}{l}\text { ns: Différences de distances moyennes entre Sans/Avec desserte TC non } \\
\text { significatives à } 5 \% \text {. }\end{array}$} \\
\hline \multicolumn{3}{|c|}{ *: Différences significatives à 5\%. } \\
\hline
\end{tabular}

La distinction des quartiers d'habitation selon leur desserte par les transports collectifs met en évidence des différences d'accessibilité dans les structures de soin de premier niveau. Dans les deux villes, vivre dans un quartier enclavé tend globalement à accroître légèrement les distances à l'équipement sanitaire de base lorsqu'il est public, plus nettement lorsqu'il est privé (700 m de plus), alors même que dans ces quartiers, très majoritairement localisés en périphérie, les possibilités d'accéder à d'autres lieux sont plus réduites du fait de la pauvreté de la desserte en transports collectifs. Nombre d'usagers du système de soins doivent ainsi se rendre loin de leur domicile, souvent à pied. Ces difficultés apparaissent également lorsque les citadins sont amenés à indiquer les problèmes rencontrés lors de la fréquentation des structures de santé.

Les problèmes d'accessibilité aux équipements publics ne se posent pas avec la même acuité pour tous les types d'offre. Un quart des ménages doualais les mentionnent pour les établissements publics, hôpitaux mais aussi centres de premier niveau pourtant censés être des services de proximité. L’insatisfaction est plus marquée à Conakry, l'état dégradé de la voirie et les très fortes difficultés de déplacement expliquant que près de deux ménages sur cinq les relèvent pour les dispensaires publics et trois sur quatre pour l'hôpital public. Les soucis d'accessibilité sont plus rarement évoqués pour l'offre privée, la situation apparaissant, là encore, moins favorable à Conakry qu'à Douala, ce qui renvoie de nouveau tant aux plus fortes difficultés de déplacement 
qu'à la moindre diffusion de ces services. Les difficultés d'accessibilité se révèlent systématiquement plus prégnantes lorsque le quartier de résidence est mal desservi par les transports collectifs, avec des proportions de ménages concernés supérieures de 8 à 20 points selon les services (figure 6).

Les dysfonctionnements propres au secteur de la santé (inégale distribution spatiale des établissements selon le niveau et le caractère public ou privé, coût trop élevé, qualité de service défaillante) contrarient l'accès des citadins aux services sanitaires, mais les difficultés pour se rendre aux centres de soins tendent à réduire encore le nombre de lieux potentiellement utilisables par les populations. Ceci est encore plus vrai pour les habitants des quartiers enclavés, qui cumulent souvent bas revenus et accès contrarié aux ressources de base.

\section{Figure 6 Part des ménages déclarant des problèmes d'accessibilité, selon la desserte du quartier de résidence par les transports collectifs (\%)}

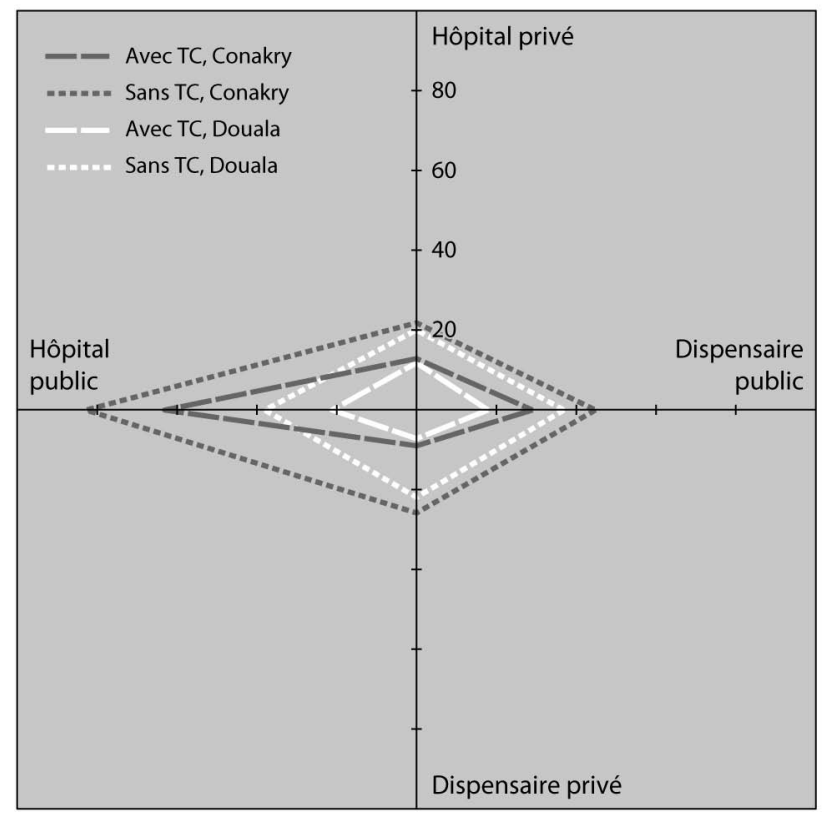

Sans desserte TC: à $15 \mathrm{mn}$ à pied ou plus de l'arrêt de transport collectif le plus proche. Avec desserte TC : à moins de $15 \mathrm{mn}$ à pied d'un arrêt de transport collectif le plus proche. Dans les deux villes, l'accessibilité est significativement (seuil de $5 \%$ ) plus citée dans les quartiers sans desserte pour tous les établissements, sauf pour les hôpitaux privés. Source: Enquêtes ménages mobilité 2003.

\section{Conclusion}

La faible part quantitative des déplacements pour la santé dans les pratiques de mobilité quotidienne ne contribue pas à en faire un objet d'étude autonome et finalisé à travers les enquêtes ménages mobilité. À titre d'exemple, une analyse comparative de la mobilité quotidienne dans les années 1990 à Bamako et Ouagadougou ne mentionnait pas ce motif de déplacement de façon autonome, placé du fait de sa rareté dans la catégorie «autre» (Diaz Olvera et al., 1998). Pourtant, les analyses menées sur Conakry et Douala apportent des éclairages originaux sur la façon dont les difficultés d'accessibilité pèsent sur le recours effectif aux soins. Elles confirment, en les précisant, les difficultés

plus grandes rencontrées dans les périphéries de différentes villes africaines, mises en évidence dans la littérature scientifique. En particulier, elles rappellent que dans le contexte de l'Afrique subsaharienne, les problèmes d'accessibilité ne se résument pas à la proximité très relative des établissements de soins et renvoient plus fortement encore à la faible motorisation des ménages, d'une part, et aux carences du secteur des transports collectifs urbains, d'autre part. 
Comme dans les secteurs de l'éducation et des transports urbains, le développement d'offres privées contribue à pallier certains manques des services publics. Pour autant, les solutions de rechange élaborées sur des critères d'efficacité bien plus que sur des critères d'équité sociospatiale sont loin d'être toutes à la portée de la majorité des citadins. L'offre privée apparaît en effet plus proche des citadins, notamment à Douala où le mouvement de diffusion de cette offre est plus avancé, mais les quartiers enclavés paraissent relativement défavorisés face à ce mouvement. Les résidants de ces quartiers se retrouvent doublement à l'écart des soins, enfermés dans une medical poverty trap (Whitehead et al., 2001), faute d'abord d'une offre de services de santé adaptée. La présence d'établissements ne signifie pas nécessairement leur utilisation, tant les dimensions coûts et qualité des prestations jouent également un grand rôle dans les possibilités effectives de recours aux services. Ce désavantage se voit redoublé et amplifié par l'inexistence ou la rareté de moyens de transport adéquats. À l'exception des cliniques et hôpitaux privés, les résidants des quartiers périphériques les moins bien desservis par les transports collectifs ont une perception particulièrement vive des problèmes d'accessibilité, pour tous les types d'établissement de soins. Ce sont aussi parmi les citadins les plus défavorisés socialement, également ceux pour qui la définition d'une offre adaptée serait la plus nécessaire.

Ces inégalités sociospatiales dans l'accès à la santé résultent pour une part de l'occultation des objectifs d'équité par les préoccupations d'efficacité dans la mise en œuvre des politiques de santé. Un tel phénomène, analysé par exemple dans le cas du Burkina Faso (Ridde, 2008), conduit à ignorer les difficultés d'accès aux soins des populations les plus défavorisées. Mais ces inégalités renvoient également à des carences similaires des politiques de transport. La planification des transports urbains repose sur des méthodes de collecte des données de déplacement privilégiant les mouvements sur des distances importantes, plutôt en heure de pointe et sur des liaisons radiales, au détriment donc des mouvements pédestres (Elson et al., 1999). Il en résulte, du fait d'une rentabilité plus élevée, une focalisation des investissements sur les infrastructures de voirie et, tout particulièrement, sur les grands axes, soit pour leur construction, soit pour leur entretien. De telles politiques bénéficient d'abord à la petite minorité des détenteurs de véhicules individuels (Vasconcellos, 2001). Elles améliorent plus marginalement la situation des usagers des transports collectifs et, au mieux, restent sans effet immédiat sur les citadins les plus pauvres ou, au pire, dégradent leurs conditions de déplacements en rendant moins sûrs les trajets pédestres.

Les enjeux de santé en milieu urbain risquent de devenir de plus en plus vifs dans les prochaines décennies, du fait du rapide accroissement démographique des aires urbaines sous la forme d'extensions périphériques "spontanées» dépourvues d’infrastructures et de services de base et toujours plus éloignées du centre. Dans ce contexte, la nécessaire adaptation de l'offre sanitaire aux besoins des populations urbaines passe non seulement par une amélioration de la qualité du service offert et par une adéquation à la solvabilité des ménages, mais aussi par la prise en compte des contraintes d'accessibilité aux différents types d'équipements, ce qui implique de prendre en compte explicitement ces impératifs dans la conception des politiques de transport. 


\section{Bibliographie}

APPARICIO, Philippe, ABDELMAJID, Mohamed, RIVA, Mylène, SHEARMUR, Richard (2008) Comparing alternative approaches to measuring the geographical accessibility of urban health services: Distance types and aggregation-error issues. International Journal of Health Geographics, vol. 7, n 7 .

BHANDERI, Miteshkumar N. et KANNAN, Srinivasan (2010) Untreated reproductive morbidities among ever married women of slums of Rajkot City, Gujarat: The role of class, distance, provider attitudes, and perceived quality of care. Journal of Urban Health, vol. $87, n^{\circ} 2$, p. 254-263.

BRUNET-JAILLY, Joseph (2001) La santé. Dans Georges P. Tapinos, Philippe Hugon et Patrice Vimard (dir.) La Côte d'Ivoire à l'aube du XXI ${ }^{e}$ siècle. Défis démographiques et développement durable. Paris, Karthala, p. 325-367.

CADOT, Emmanuelle et HARANG, Maud (2006) Offre de soins et expansion urbaine, conséquences pour l'accès aux soins. L'exemple de Ouagadougou (Burkina Faso). Espace Populations Sociétés, n² 2-3, p. 329-339.

COMMEYRAS, Christophe, NDO, Jean R., MERABET, Omar, KONÉ, Hamidou et RAKOTONDRABÉ, Faraniaina P. (2006) Comportement de recours aux soins et aux médicaments au Cameroun. Cahiers Santé, vol. $16, \mathrm{n}^{\circ} 1$, p. 5-12.

COMOLET, Thierry (2000) La santé en Guinée: un modèle de système de soins de santé primaires à revisiter. Médecine tropicale, vol. $60, \mathrm{n}^{\circ} 1$, p. 25-28.

DEVElay, Aude, SAUERBorn, Rainer et DIESFELD, Hans J. (1996) Utilization of health care in an African urban area: results from a household survey in Ouagadougou, Burkina Faso. Social Science \& Medicine, vol. 43, $\mathrm{n}^{\circ}$ 11, p. 1611-1619.

DIAZ OLVERA, Lourdes, PLAT, Didier et POCHET, Pascal (1998) Villes africaines au quotidien. Mobilités quotidiennes et contraintes sociales à Bamako et Ouagadougou. Lyon, LET.
DIAZ OLVERA, Lourdes, PLAT, Didier et POCHET, Pascal (2007) Mobilité quotidienne en temps de crise. Belgeo - Revue belge de géographie, $\mathrm{n}^{\circ}$ 2, p. 173-187.

DIAZ OLVERA, Lourdes, PLAT, Didier et POCHET, Pascal (2008) Household transport expenditure in Sub-Saharan African cities: Measurement and analysis. Journal of Transport Geography, vol. 16, $\mathrm{n}^{\circ} 1$, p. 1-13.

DIAZ OLVERA, Lourdes, PLAT, Didier et POCHET, Pascal (2010) À l'écart de l'école? Pauvreté, accessibilité et scolarisation à Conakry. Revue Tiers Monde, ${ }^{\circ} 202$, p. 167-183.

DOUMBOUYA, Mohamed L. (2008) Accessibilité des services de santé en Afrique de l'Ouest: le cas de la Guinée. LEFI, Université Lumière Lyon 2, Working Paper $n^{\circ} 2008-2$. [En ligne]. http://hal. archives-ouvertes.fr/docs/00/22/96/96/ PDF/WPDoumbouya.pdf

DRAMÉ, Fatou M. (2006) Attraction des services de soins de santé primaires et des centres de dépistage du VIH à Dakar (Sénégal). Améliorer l'approche par zone de polarisation. Espace Populations Sociétés, $\mathrm{n}^{\circ} 2-3$, p. 361-371.

ELSON, Diane, EVERS, Barbara et TURNER, Jeff (1999) Transport sector programmes in developing countries: Integrating a gender analysis. University of Manchester, Graduate School of Social Sciences.

ESER, Erhan, DINÇ, Gönül, ORAL, Ahmet M. et ÖZCAN, Cemil (2005) Contrasting children and women's health and the determinants of health in a small-sized city. Journal of Urban Health, vol. 82, $n^{\circ} 4$, p. 666-681.

ESSENDI, Hildah, MILLS, Samuel et FOTSO, Jean-Christophe (2010) Barriers to formal emergency obstetric care services' utilization. Journal of Urban Health, vol. 88, suppl. 2, p. S357-S369.

FALL, Abdou S. (2007) Bricoler pour survivre. Perceptions de la pauvreté dans l'agglomération urbaine de Dakar. Paris, Karthala. 
FOTSO, Jean-Christophe, EZEH, Alex et ORONJE, Rose (2008) Provision and use of maternal health services among urban poor women in Kenya: What do we know and what can we do? Journal of Urban Health, vol. 85, n³, p. 428-442.

GAUTHIER, Bernard et WANE, Waly (2008) Bypassing health providers: the quest for better price and quality of health care in Chad. World Bank, Policy Research Working Paper, $\mathrm{n}^{\circ}$ WPS 4462.

GAUVRIT, Éric et OKALLA, Raphaël (2001) La difficile mise en place des districts sanitaires urbains. Bulletin de l'APAD, Un système de santé en mutation: le cas du Cameroun, $\mathrm{n}^{\circ}$ 21. [En ligne.] http://apad. revues.org/sommaire25.html

GOBBERS, Didier (2004) La coopération en santé dans les pays africains. Revue Actualité et dossier en santé publique, $\mathrm{n}^{\circ} 48$, p. 8-13. [En ligne.] http://www.bdsp. ehesp.fr/fulltext/Show.asp?Ref=315812

GROUPE HUIT-BCEOM (2003) Étude de faisabilité d'un programme prioritaire d'aménagement des quartiers sous-équipés de Conakry. Rapport de phase 1. Direction Nationale de l'aménagement du territoire et de l'urbanisme du ministère de l'Urbanisme et de l'Habitat de la République de Guinée.

GUAGLIARDO, Mark F. (2004) Spatial accessibility of primary care: concepts, methods and challenges. International Journal of Health Geographics, vol. 3, n 3 .

HARPHAM, Trudy (2009) Urban health in developing countries: what do we know and where do we go? Health \& Place, vol. 15, $\mathrm{n}^{\circ} 1$, p. 107-116.

JAFFRÉ, Yves (2004) Prolégomènes à une réforme des services de santé: de l'identification des dysfonctionnements à la définition d'outils efficaces. Médecine Tropicale, vol. 64, n ${ }^{\circ}$, p. 527-532.

KINDA, Fatoumata (1987) Ménages populaires à Ouagadougou. Université de Nantes, Thèse de doctorat en sociologie.
LÉVY, Jacques et LUSSAULT, Michel (2003) Dictionnaire de la géographie et de l'espace des sociétés. Paris, Belin.

MAMADANI, Masuma et BANGSER, Maggie (2004) Poor people's experiences of health services in Tanzania: A literature review. Reproductive Health Matters, vol. 12, n 24 , p. $138-153$.

MBARA, Tatenda C. (2002) Activity patterns, transport and policies for the urban poor in Harare, Zimbabwe. Final country report. [En ligne.] www.transport-links. org/transport_links/filearea/documentstore/305_Zimbabwe\%20Final\%20Report\%20-\%20Final.PDF

MÉDARD, Jean-François (2001) Décentralisation du système de santé publique et ressources humaines au Cameroun. Bulletin de l'APAD, Un système de santé en mutation: le cas du Cameroun, $\mathrm{n}^{\circ} 21$. [En ligne.] http://apad.revues.org/sommaire25.html

MORE, Neena S., ALCOCK, Glyn, DAS, Sushmita, BAPAT, Ujwala, JOSHI, Wasundhara et OSRIN, David (2011) Spoilt for choice? Cross-sectional study of care-seeking for health problems during pregnancy in Mumbai slums. Global Public Health, vol. 6, n 7 , p. 746-759.

OKALLA, Raphaël et LE VIGOUROUX, Alain (2001) Cameroun: de la réorientation des soins de santé primaires au plan national de développement sanitaire. Bulletin de l'APAD, Un système de santé en mutation: le cas du Cameroun, $\mathrm{n}^{\circ} 21$. [En ligne.] http://apad.revues.org/sommaire25.html

OLIVIER DE SARDAN, Jean-Pierre, BAKO-ARIFARI, Nassirou et MOUMOUNI, Adamou (2007) La corruption dans le domaine de la santé. Dans Giorgio Blundo et JeanPierre Olivier de Sardan (dir.) État et corruption en Afrique. Paris, APAD-Karthala, p. 225-247.

OMS (2010) National Health Accounts. [En ligne.] http://www.who.int/nha/country/ en/index.html 
PAEZ, Antonio, MERCADO, Ruben G., FARBER, Steven, MORENCY, Catherine et ROORDA, Matthew (2010) Accessibility to health care facilities in Montreal Island: an application of relative accessibility indicators from the perspective of senior and non-senior residents. International Journal of Health Geographics, vol. 9, n 52.

PENCHANSKY, Roy et THOMAS, William J. (1981) The concept of access: Definition and relationship to consumer satisfaction. Medical Care, vol. 19, $n^{\circ}$ 2, p. 127-140.

RAUX, Charles, MERCIER Aurélie et OVTRACHT, Nicolas (2008) Evaluation économique des politiques de transport et indicateurs d'accessibilité spatiale: l'apport des SIG. Cybergeo: European Journal of Geography, $\mathrm{n}^{\circ} 435,11 / / 11 / 2008$, [En ligne.] http://cybergeo.revues. org/21333.

REBOUHA, Fafa (2007) Concentration des services de santé, contraintes de mobilité et difficultés d'accès aux soins dans la métropole d'Oran. Territoire en Mouvement, $\mathrm{n}^{\circ} 4$, p. 34-46.

RIDDE, Valéry (2005) Politiques publiques de santé et équité en Afrique de l'Ouest. Le cas de l'Initiative de Bamako au Burkina Faso. Faculté de médecine, Faculté des sciences infirmières, Université Laval, $\mathrm{PhD}$, Thèse de doctorat en santé communautaire. [En ligne.] http://archimede.bibl.ulaval.ca/ archimede/fichiers/23020/23020.html

RIDDE, Valéry (2008) The problem of the worst-off is dealt with after all other issues: The equity and health policy implementation gap in Burkina Faso. Social Science \& Medicine, vol. 66, $\mathrm{n}^{\circ}$ 6, p. $1368-1378$.

SAHN, David E. et STIFEL, David C. (2003) Progress Toward the Millennium Development Goals in Africa. World Development, vol. 31, $\mathrm{n}^{\circ} 1$, p. 23-52.

SALEM, Gérard (1998) La santé dans la ville. Géographie d'un petit espace dense: Pikine (Sénégal). Paris, Karthala-ORSTOM.
SALZE, Paul, BANOS, Arnaud, OPPERT, JeanMichel, CHARREIRE, Hélène, CASEY, Romain, SIMON, Chantal, CHAIX, Basile, BADARIOTTI, Dominique et WEBER, Christiane (2011) Estimating spatial accessibility to facilities on the regional scale: an extended commuting-based interaction potential model. International Journal of Health Geographics, vol. 10, nº 2 .

SASAKI, Satoshi, FUJINO, Yasuyuki, IGARASHI, Kumiko, TANABE, Naohito, MULEYA, Clara M., SUZUKI, Hiroshi (2010) Access to a health facility and care-seeking for danger signs in children: before and after a community-based intervention in Lusaka, Zambia. Tropical Medicine \& International Health, vol. 15, $\mathrm{n}^{\circ}$ 3, p. 312-320.

SHERMAN, Jill E., SPENCER, John, PREISSER, John S., GESLER, Wilbert M. et ARCURY, Thomas A. (2005) A suite of methods for representing activity space in a healthcare accessibility study. International Journal of Health Geographics, vol. 4, n² 24.

SITRASS (2004a) Pauvreté et mobilité urbaine à Douala. Rapport final pour la Banque Mondiale dans le cadre du SSATP. Lyon, SITRASS. [En ligne.] http://www4. worldbank.org/afr/ssatp/Resources/ PapersNotes/Douala.pdf.

SITRASS (2004b) Pauvreté et mobilité urbaine à Conakry. Rapport final pour la Banque Mondiale dans le cadre du SSATP. Lyon, SITRASS. [En ligne.] http://www4. worldbank.org/afr/ssatp/Resources/ PapersNotes /Conakry.pdf.

TALEN, Emily et ANSELIN, Luc (1998), Assessing spatial equity: an evaluation of measures of accessibility to public playgrounds. Environment and Planning Part. A, vol. 30, n 4, p. 595-613.

VASCONCELLOS, Eduardo A. (2001) Urban transport, environment and equity. The case for developing countries. Londres, Earthscan.

WHITEHEAD, Margaret, DAHLGREN, Göran et EVANS, Timothy (2001) Equity and health sector reforms: can low-income countries escape the medical poverty trap? The Lancet, vol. 358, n ${ }^{\circ}$ 9284, p. 833-836. 